\title{
ON POLAR RELATIONS OF ABSTRACT HOMOGENEOUS POLYNOMIALS
}

\author{
BY
}

\author{
NEYAMAT ZAHEER( ${ }^{(1)}$
}

ABSTRACT. In this paper we generalize, to vector spaces over algebraically closed fields of characteristic zero, two well-known classical results due to Laguerre and Grace, concerning, respectively, the relative location of the zeros of a complex-valued polynomial and its polar-derivative and the relative location of the zeros of two apolar polynomials. Vector space analogues of their results were generalized, to a certain degree, by Hörmander, Marden, and Zervos. Our results in this paper further generalize their results and, in the complex plane, improve upon those of Laguerre and Grace. Besides, the present treatment unifies their completely independent approaches into an improved and more systematic and abstract theory. We have also shown that our results are best possible in the sense that they cannot be further generalized in certain directions.

1. Introduction. Let $E$ be a vector space over a field $K$ of characteristic zero. A mapping $P$ from $E$ to $K$ is called [9] , [8, p. 55], [5, pp. 760-763] an abstract homogeneous polynomial (a.h.p.) of degree $n$ if for every $x, y$ in $E$,

$$
P(s x+t y)=\sum_{k=0}^{n} A_{k}(x, y) s^{k} t^{n-k} \quad \forall s, t \in K,
$$

where the coefficients $A_{k}(x, y)$ are elements of $K$ which are independent of $s$ and $t$ and depend only on $x$ and $y$. Let $\mathbf{P}_{n}$ denote the class of all $n$ th-degree a.h.p.'s from $E$ to $K$. The $n$ th-polar of $P$ is the mapping (see [6, Lemma 1] for its existence and uniqueness) $P\left(x_{1}, x_{2}, \ldots, x_{n}\right)$ from $E \times E \times \ldots \times E$ to $K$ which is linear in each $x_{k}$ and symmetric in the set $\left\{x_{k}\right\}$ such that $P(x, x, \ldots x)$ $=P(x)$ for every $x$ in $E$. We may then specify the $k$ th-polar of $P$ by the relation

$$
P\left(x_{1}, \ldots, x_{k}, x\right)=P\left(x_{1}, \ldots, x_{k}, x, x, \ldots, x\right) .
$$

Presented to the Society, January 18, 1972 under the title Null-sets of abstract homogeneous polynomials in vector spaces; received by the editors May 30,1973 and, in revised form, November 20, 1974.

AMS (MOS) subject classifications (1970). Primary 30A08; Secondary 12D10.

Key words and phrases. Abstract homogeneous polynomials and their polars, apolar polynomials, circular cones, hermitian cones, generalized circular regions, and generalized balls.

(1) The results in this paper are partly contained in the author's doctoral dissertation (1971) at the University of Wisconsin-Milwaukee under the supervision of the University of Wisconsin-Milwaukee Distinguished Professor Morris Marden. The author wishes to thank him for his useful suggestions and advice all along. 
If $P \in \mathbf{P}_{n}$, the null-set $Z_{P}(x, y)$ of $P$ (relative to given elements $x, y$ of $E$ ) is defined by

$$
Z_{P}(x, y)=\{s x+t y \neq 0 \mid s, t \in K ; P(s x+t y)=0\}
$$

From now on we shall assume that $K$ is an algebraically closed field of characteristic zero. As is well known [6] (see also [1, pp. 38-40], [12, pp. 248-255]), such a field has a maximal ordered ("real closed" according to van der Waerden) subfield $K_{0}$ such that $K=K_{0}(i)$, where $-i^{2}$ is the unit element of $K$. Since every element $z$ in $K$ has the form $z=a+i b$ with $a, b$ in $K_{0}$, we define $\bar{z}=a-i b, \operatorname{Re}(z)=(z+\bar{z}) / 2$, and $|z|=\left(a^{2}+b^{2}\right)^{1 / 2}$. A subset $A$ of $K$ is called $K_{0}$-convex if $\Sigma_{j=1}^{n} \mu_{j} a_{j} \in A$ for every $a_{j}$ in $A$ and $\mu_{j}$ in $K_{0+}$ (the set of all nonnegative elements of $K_{0}$ ) such that $\sum_{j=1}^{n} \mu_{j}=1$. Adjoin to $K$ an element $\omega$ (called infinity) and furnish $K \cup\{\omega\}$ (denoted $K_{\omega}$ ) with the following structure: (1) the subset $K$ of $K_{\omega}$ preserves its initial field structure; and (2) $a$ $+\omega=\omega+a=\omega$ for every $a$ in $K, a \cdot \omega=\omega \cdot a=\omega$ for every $a \in K-\{0\}$, and $\omega^{-1}=0,0^{-1}=\omega$. Given an element $\zeta$ of $K$, we define

$$
\varphi_{\zeta}(z)=1 /(z-\zeta) \quad \forall z \in K_{\omega}
$$

A subset $A$ of $K_{\omega}$ is called [16, pp. 353-373] , [15, pp. 25-26] a generalized circular region of $K_{\omega}$ if either $A$ is one of the sets $\varnothing, K, K_{\omega}$, or $A$ satisfies the following two conditions:

(1) $\varphi_{\zeta}(A)$ is $K_{0}$-convex for every $\zeta \in K-A$;

(2) $\omega \in A$ if $A$ is not $K_{0}$-convex.

$D\left(K_{\omega}\right)$ denotes the class of all generalized circular regions of $K_{\omega}$. The empty set $\varnothing, K, K_{\omega}$, and single-point sets (and their complements in $K_{\omega}$ ) are trivial generalized circular regions of $K_{\omega}$. The characterization (due to Zervos [16, pp. 372-387]) of the class $D\left(K_{\omega}\right)$, when $K$ is the field $\mathbf{C}$ of complex numbers, leads to the following result $[16, \mathrm{p} .352]$ : The nontrivial generalized circular regions of $\mathbf{C}_{\omega}$ are the open interior (or, exterior) of circles or the half-planes, adjoined with a connected subset (possibly empty) of their boundary. Generalized circular regions of $\mathbf{C}_{\omega}$, with all or no boundary points included, will be termed as (classical) circular regions of $\mathbf{C}_{\boldsymbol{\omega}}$.

The class of generalized balls of $K_{\omega}$ consists of the following subsets of $K_{\omega}$ :

(1) $\varnothing, K, K_{\omega}$, and the sets of the type $\{z \in K \mid \alpha z \bar{z}+\beta z+\bar{\beta} \bar{z}+\gamma \leqslant 0\}$ or $\{z \in K \mid \alpha z \bar{z}+\beta z+\bar{\beta} \bar{z}+\gamma<0\}$ for some $\alpha \in K_{0+}, \gamma \in K_{0}$, and $\beta \in K$; and

(2) the complements in $K_{\omega}$ of the sets in (1).

It is trivial to show that generalized balls are generalized circular regions of $K_{\omega}$. 
2. Circular cones. In this section we give some definitions and concepts leading to the construction of certain subsets (called circular cones $[15, \mathrm{pp} .36-$ 40]) of a vector space $E$ over $K$ and we prove some results which will be frequently used in our work. Throughout this paper $E^{2}$ denotes $E \times E$ and $L[x, y]$ the subspace of $E$ generated by the elements $x$ and $y$ of $E$. We define an equivalence relation " $\sim$ " on $E^{2}$ as follows: We say that $\left(x_{1}, y_{1}\right) \sim\left(x_{2}, y_{2}\right)$ if and only if $L\left[x_{1}, y_{1}\right]=L\left[x_{2}, y_{2}\right]$. The equivalence class $[(x, y)]$, containing the element $(x, y)$ of $E^{2}$, is called nontrivial if $x$ and $y$ are linearly independent (trivial otherwise). By axiom of choice we choose a unique element from each nontrivial equivalence class and call the set $N\left(\subseteq E^{2}\right)$ of elements thus chosen as a nucleus of $E^{2}$. Note that $N$ is nonempty if the dimension of $E$ is at least two. We define a mapping $G$ from $N$ into $D\left(K_{\omega}\right)$ as a circular mapping. We then define a mapping $T_{G}$ (for a given $G$ ) from $N$ into the class of all subsets of $E$ by

$$
T_{G}(x, y)=\{s x+t y \neq 0 \mid s, t \in K ; s / t \in G(x, y)\} .
$$

Finally, given a nucleus $N$ of $E^{2}$ and a circular mapping $G$ from $N$ into $D\left(K_{\omega}\right)$, we define the subset $E_{0}(N, G)$ of $E$ by

$$
E_{0}(N, G)=\bigcup T_{G}(x, y)
$$

and call it a circular cone in $E$ relative to $N$ and $G$. (The union in (2.2) is taken over all elements $(x, y) \in N$.)

REMARK (2.1). In a two-dimensional vector space $E$, every cone $E_{0}(N, G)$ is of the form

$$
E_{0}(N, G)=\left\{s x_{0}+t y_{0} \neq 0 \mid s, t \in K ; s / t \in A\right\}
$$

for some $A \in D\left(K_{\omega}\right)$, where $x_{0}, y_{0}$ are any two linearly independent elements of $E, N=\left\{\left(x_{0}, y_{0}\right)\right\}$, and $G\left(x_{0}, y_{0}\right)=A$. Now we describe the class of sets which were used by Hörmander [6] and Marden [10] for their generalizations of the theorems of Laguerre [7] and Grace [4] respectively. The sets of this class, which we term as hermitian cones, are subsets $E_{1}$ of $E$ of the form $E_{1}=$ $\{x \neq 0 \mid H(x, x) \geqslant 0\}$ (or the ones obtained by replacing in this expression the inequality " $\geqslant$ " by " $<$ ", " $>$ ", or " $<$ "), where $H(x, y)$ is a hermitian symmetric form [9] defined from $E^{2}$ to $K$. The following proposition shows that every hermitian cone is necessarily a circular cone.

Proposition (2.2). Let $E_{1}$ be a hermitian cone in $E$. Given a nucleus $N$ of $E^{2}$, there exists a circular mapping $G$ from $N$ into $D\left(K_{\omega}\right)$ such that the circular cone $E_{0}(N, G)=E_{1}$ and $E_{1} \cap L[x, y]=T_{G}(x, y)$ for every $(x, y) \in N$, where $T_{G}$ is as defined by (2.1). 
Proof. We prove this proposition for the case when the hermitian cone $E_{1}$ is given by $E_{1}=\{x \in E \mid x \neq 0 ; H(x, x)<0\}$. The proofs for the remaining three cases are similar. If $(x, y)$ belongs to $N$, then $x$ and $y$ are linearly independent and

$$
\begin{aligned}
E_{1} \cap L[x, y] & =\{s x+t y \neq 0 \mid H(s x+t y, s x+t y)<0\} \\
& =\{s x+t y \neq 0 \mid \alpha s \bar{s}+\beta s \bar{t}+\bar{\beta} \bar{s} t+\gamma t \bar{t}<0\}
\end{aligned}
$$

where $\alpha=H(x, x), \beta=H(x, y)$, and $\gamma=H(y, y)$. We now construct a circular mapping $G$ from $N$ into $D\left(K_{\omega}\right)$ as follows: For every $(x, y) \in N$, define

$$
\begin{array}{ll}
G(x, y)=\{\rho \in K \mid \alpha \rho \bar{\rho}+\beta \rho+\bar{\beta} \bar{\rho}+\gamma<0\} & \text { if } x \notin E_{1}, \\
G(x, y)=\{\rho \in K \mid \alpha \rho \bar{\rho}+\beta \rho+\bar{\beta} \bar{\rho}+\gamma<0\} \cup\{\omega\} & \text { if } x \in E_{1} .
\end{array}
$$

It is easy to verify that $E_{1} \cap L[x, y]=T_{G}(x, y)$ for every $(x, y) \in N$. Consequently, the circular cone $E_{0}(N, G)$ coincides with $E_{1}$. This completes our proof.

The following example shows that there do exist circular cones which are not hermitian cones.

EXAMPLE (2.3). Let $H$ be a hermitian symmetric form having a Lorentz signature [6, pp. 58-59], then $[6$, p. 58] there exist linearly independent elements $x_{0}, y_{0}$ of $E$ such that $H\left(x_{0}, x_{0}\right)=1, H\left(x_{0}, y_{0}\right)=0$, and $H\left(y_{0}, y_{0}\right)<0$. Now we choose a nucleus $N$ of $E^{2}$ such that $\left(x_{0}, y_{0}\right) \in N$ (which is always possible). Let $E_{1}=\{x \in E \mid H(x, x)<0\}$. By Proposition (2.2), there exists a circular cone $E_{0}(N, G)$ such that $E_{0}(N, G)=E_{1}$. Note that each set $G(x, y)$ in (2.3) is a generalized ball (with no boundary point included in it) of $K_{\omega}$ $\left(\cong K_{0 \omega}^{2}\right)$ and, hence, a generalized circular region of $K_{0 \omega}^{2}$. Since (cf. (2.3))

$$
G\left(x_{0}, y_{0}\right)=\left\{\left.\rho \in K|| \rho\right|^{2}<-H\left(y_{0}, y_{0}\right)\right\} \text {, }
$$

we see that $G\left(x_{0}, y_{0}\right)$ has at least three boundary points (see [16, Theorem 2, p. 381]). We adjoin to $G\left(x_{0}, y_{0}\right)$ a suitable nonempty proper subset of its boundary (e.g., a single-point set) so that the resulting set $A$ (say) becomes a generalized circular region different from a generalized ball. Now we define another circular mapping $G_{0}$ from $N$ into $D\left(K_{\omega}\right)$ which agrees with $G$ everywhere except at $\left(x_{0}, y_{0}\right)$, where we define $G_{0}\left(x_{0}, y_{0}\right)=A$. Then $E_{0}\left(N, G_{0}\right)$ is a circular cone (containing $E_{1}$ ) which is not a hermitian cone. For otherwise, by Proposition (2.2), there would exist a circular mapping (via (2.3)) $G_{1}$ (say) from $N$ into $D\left(K_{\omega}\right)$ such that

$$
T_{G_{0}}\left(x_{0}, y_{0}\right)=E_{0}\left(N, G_{0}\right) \cap L\left[x_{0}, y_{0}\right]=T_{G_{1}}\left(x_{0}, y_{0}\right) \text {. }
$$

This would then imply that $A=G_{1}\left(x_{0}, y_{0}\right)$, which contradicts the fact that 
$G_{1}\left(x_{0}, y_{0}\right)$ is a generalized ball (cf. (2.3)). Hence $E_{0}\left(N, G_{0}\right)$ is a circular cone which is not a hermitian cone.

REMARK. This method of construction suggests the existence of a large class (at least as large as the class of all hermitian cones defined by hermitian symmetric forms of Lorentz signature) of circular cones $E_{0}\left(N, G_{0}\right)$ different from hermitian cones.

In the light of Example (2.3), we now state the following

THEOREM (2.4). The class of all circular cones in $E$ contains properly the class of all hermitian cones in $E$.

3. A generalization of Laguerre's theorem. Our main result of this section uses circular cones and is expressed in the following theorem which generalizes the results of Laguerre [8, Theorem (13.2)], Hörmander [6, Theorem 1] (cf. also Marden [8, Theorem (14.1)]), and Zervos [16, Corollary (4.2), p. 360]. We assume that the dimension of $E$ is at least two.

THEOREM (3.1). If $P \in \mathrm{P}_{n}$ and $E_{0}(N, G)$ is a circular cone in $E$ such that $Z_{p}(x, y) \subseteq T_{G}(x, y)$ for every $(x, y) \in N$, then $P\left(x_{1}, x, \ldots, x\right) \neq 0$ for all nonzero elements $x$ and $x_{1}$ in $E-E_{0}(N, G)$.

Proof. Let $E_{0}=E_{0}(N, G)$ and $x, x_{1}$ be any two nonzero elements of $E-E_{0}$, so that $x, x_{1} \notin T_{G}\left(x_{0}, y_{0}\right)$ for any $\left(x_{0}, y_{0}\right) \in N$. If $x, x_{1}$ are linearly dependent, then $x_{1}=\alpha x$ for some $\alpha \in K-\{0\}$. Consequently, $P\left(x_{1}, x, \ldots, x\right)$ $=\alpha \cdot P(x) \neq 0$. For, the vanishing of $P(x)$ would contradict the fact that $x \in$ $E-E_{0}$.

If $x$ and $x_{1}$ are linearly independent, then there exists an element $\left(x_{0}, y_{0}\right)$ $\in N$ such that $x=\alpha x_{0}+\beta y_{0}$ and $x_{1}=\alpha_{1} x_{0}+\beta_{1} y_{0}$ for some elements $\alpha, \beta$, $\alpha_{1}, \beta_{1}$ of $K$ for which $\alpha \beta_{1}-\alpha_{1} \beta \neq 0$. Since $K$ is algebraically closed, we can write

$$
P\left(s x+t x_{1}\right)=\prod_{j=1}^{n}\left(\delta_{j} s-\gamma_{j} t\right) \quad \forall s, t \in K,
$$

where the elements $\delta_{j}$ and $\gamma_{j}$ of $K$ depend only on $x, x_{1}$ and are independent of $s$ and $t$. Since $P\left(\gamma_{j} x+\delta_{j} x_{1}\right)=0$ for all $j$, we see that

$$
\gamma_{j} x+\delta_{j} x_{1}=\left(\gamma_{j} \alpha+\delta_{j} \alpha_{1}\right) x_{0}+\left(\gamma_{j} \beta+\delta_{j} \beta_{1}\right) y_{0} \in T_{G}\left(x_{0}, y_{0}\right)
$$

and that

$$
\left(\gamma_{j} \alpha+\delta_{j} \alpha_{1}\right) /\left(\gamma_{j} \beta+\delta_{j} \beta_{1}\right) \in G\left(x_{0}, y_{0}\right) \quad \forall j=1,2, \ldots, n .
$$

Now

$$
P(x)=\delta_{1} \delta_{2} \ldots \delta_{n} \neq 0, \quad P\left(x_{1}\right)=(-1)^{n} \gamma_{1} \gamma_{2} \ldots \gamma_{n} \neq 0
$$


and consequently $\gamma_{j}, \delta_{j} \neq 0$ for all $j$. If $\rho_{j}=\gamma_{j} / \delta_{j}$, then $\rho_{j} \neq 0, \omega$ for all $j$. Therefore (due to (3.1))

$$
\rho_{j}^{\prime}=\left(\rho_{j} \alpha+\alpha_{1}\right) /\left(\rho_{j} \beta+\beta_{1}\right) \in G\left(x_{0}, y_{0}\right) \text { for all } j .
$$

Let $U$ be the homographic transformation [16, p. 353] of $K_{\omega}$ given by

$$
U(\rho)=\left(\rho \beta_{1}-\alpha_{1}\right) /(-\rho \beta+\alpha) \text { for all } \rho \in K_{\omega} \text {. }
$$

Then (3.2) implies that $\rho_{j}=U\left(\rho_{j}^{\prime}\right) \in U\left(G\left(x_{0}, y_{0}\right)\right)$ for all $j$. Since $x, x_{1} \notin E_{0}$, obviously $\alpha / \beta$ and $\alpha_{1} / \beta_{1}$ do not belong to $G\left(x_{0}, y_{0}\right)$ and so $0, \omega \notin U\left(G\left(x_{0}, y_{0}\right)\right)$. By using the definition of a generalized circular region, we know $[16$, p. 353] that $U\left(G\left(x_{0}, y_{0}\right)\right)$ is a $K_{0}$-convex subset of $K$ not containing the element 0 . Therefore

$$
\frac{1}{n} \cdot \sum_{j=1}^{n} \rho_{j} \neq 0
$$

But we know $[6$, p. 57] that

$$
P\left(x_{1}, x, \ldots, x\right)=-\frac{1}{n} \cdot \sum_{j=1}^{n} \rho_{j} .
$$

Now (3.3) completes the proof of Theorem (3.1).

Successive applications of Theorem (3.1) for hermitian cones (a proper subfamily of circular cones) give the following result.

Corollary (3.2) (Hörmander [6, Theorem 1]). Let $P \in \mathrm{P}_{n}$ and $E_{1}$ be a hermitian cone defined by

$$
E_{1}=\{x \in E \mid x \neq 0 ; H(x, x) \geqslant 0\},
$$

where $H$ is a hermitian symmetric form from $E^{2}$ to $K$. If $P(x) \neq 0$ for every $x \in E_{1}$, then $P\left(x_{1}, x_{2}, \ldots, x_{n}\right) \neq 0$ for every $x_{1}, x_{2}, \ldots, x_{n} \in E_{1}$.

Proof. Let $N$ be a nucleus of $E^{2}$. Since $E-E_{1} \cup\{0\}$ is also a hermitian cone, Proposition (2.2) implies the existence of a circular mapping $G$ (given by (2.3)) such that $E-E_{1} \cup\{0\}=E_{0}(N, G)$ and

$$
\left(E-E_{1} \cup\{0\}\right) \cap L[x, y]=T_{G}(x, y) \quad \forall(x, y) \in N .
$$

Clearly, then $Z_{P}(x, y) \subseteq T_{G}(x, y)$ for every $(x, y) \in N$ and the proof of Corollary (3.2) is obvious by successively applying Theorem (3.1) to $P$ and its successive polars.

Second application of Theorem (3.1) leads to the following corollary which is an improvement upon a classical theorem of Laguerre (see [8, Theorem (13.2)]), in the sense that we use generalized circular regions of $\mathbf{C}_{\omega}$ in our result while 
Laguerre used the (classical) circular regions in his theorem.

COROLlARY (3.3). Let $f(z)=\sum_{k=0}^{n} a_{k} z^{k}$ be an nth-degree polynomial from C to C. Given the elements $\zeta_{1}, \ldots, \zeta_{k}(k \leqslant n-1)$ in $\mathbf{C}$, we define the sequence $f_{k}(z)$ (more precisely $f_{k}\left(\zeta_{1}, \ldots, \zeta_{k}, z\right)$ ) of polar-derivatives of $f$ by

$$
f_{k}(z)=(n-k+1) f_{k-1}(z)+\left(\zeta_{k}-z\right) f_{k-1}^{\prime}(z),
$$

where $f_{0}(z)=f(z)$. If all the zeros of $f$ lie in a generalized circular region $C$ of $\mathrm{C}_{\omega}$, then (for each $k$ )

$$
f_{k}\left(\zeta_{1}, \ldots, \zeta_{k}, z\right) \neq 0 \quad \forall \zeta_{1}, \ldots, \zeta_{k}, z \notin C .
$$

Proof. Any element $x$ in $C^{2}$ can be uniquely written as $x=s x_{0}+t y_{0}=$ $(s, t)$ for some $s, t \in \mathrm{C}$, where $x_{0}=(1,0)$ and $y_{0}=(0,1)$. Then (cf. Remark (2.1)) the set

$$
E_{0}(N, G)=\left\{s x_{0}+t y_{0} \neq 0 \mid s, t \in \mathrm{C} ; s / t \in C\right\}
$$

is a circular cone in $\mathrm{C}^{2}$, where $N=\left\{\left(x_{0}, y_{0}\right)\right\}$ and $G\left(x_{0}, y_{0}\right)=C$. Define an $n$ th-degree a.h.p. from $\mathrm{C}^{2}$ to $\mathrm{C}$ by

$$
P(x) \equiv P\left(s x_{0}+t y_{0}\right)=\sum_{k=0}^{n} a_{k} s^{k} t^{n-k} \quad \forall x=(s, t) \in \mathrm{C}^{2}
$$

Setting $z=s / t, \zeta_{j}=s_{j} / t_{j}$, and $x_{j}=\left(s_{j}, t_{j}\right)$, we observe that (cf. [8, p. 45] and $[8$, equation (14.6), p. 56])

$$
P(x)=t^{n} f(z)
$$

and

$$
\begin{aligned}
P\left(x_{1}, \ldots, x_{k}, x, \ldots, x\right) & \\
& =[(n-k) ! / n !] \cdot t_{1} \cdots t_{k} \cdot t^{n-k} f_{k}\left(\zeta_{1}, \ldots, \zeta_{k}, z\right),
\end{aligned}
$$

for all nonzero elements $x_{1}, \ldots, x_{k}, x$ of $\mathbf{C}^{2}$. Consequently, $Z_{P}\left(x_{0}, y_{0}\right) \subseteq$ $T_{G}\left(x_{0}, y_{0}\right)=E_{0}(N, G)$ and the proof immediately follows from successive applications of Theorem (3.1).

Our last application of Theorem (3.1) leads to the following corollary, a result due to Zervos [16, Corollary (4.2), p. 360].

COROllary (3.4). Let $f(z)=\Sigma_{k=0}^{n} a_{k} z^{k}$ be an nth-degree polynomial from $K$ to $K$ and let $f^{\prime}(z)=\Sigma_{k=1}^{n} k a_{k} z^{k-1}$. Given the elements $\zeta_{1}, \ldots, \zeta_{k}(k \leqslant$ $n-1$ ) in $K$, we define the sequence $f_{k}(z)$ (more precisely $f_{k}\left(\zeta_{1}, \ldots, \zeta_{k}, z\right)$ ) of polar-derivatives of $f$ by

$$
f_{k}(z)=(n-k+1) f_{k-1}(z)+\left(\zeta_{k}-z\right) f_{k-1}^{\prime}(z),
$$


where $f_{0}(z)=f(z)$. If all the zeros of $f$ lie in a generalized circular region $C$ of $K_{\omega}$, then (for each $k$ ) $f_{k}\left(\zeta_{1}, \ldots, \zeta_{k}, z\right) \neq 0 \forall \zeta_{1}, \ldots, \zeta_{k}, z \notin C$.

Proof. The proof of this corollary is exactly the same as that of Corollary (3.3), when $\mathbf{C}$ is replaced by $K$.

The following example shows that Theorem (3.1) is best possible in the sense that it cannot be generalized for vector spaces over nonalgebraically closed fields of characteristic zero.

ExAmple (3.5). Let $K_{0}$ be a maximal ordered field (so that $K_{0}$ is a nonalgebraically closed field of characteristic zero $[7, \mathrm{pp} .233,250])$. Take $E=K_{0}^{2}$ with a basis consisting of elements $x_{0}=(1,0)$ and $y_{0}=(0,1)$. Let $C$ be the generalized circular region of $K_{0}$ given by $C=\{-1\}$. (Though we have defined the generalized circular regions for an algebraically closed field of characteristic zero, but the definition remains the same for a general field as in [16, pp. 353, $373]$ or $[15$, p. 26] .) Then (cf. Remark (2.1)) the set

$$
E_{0}(N, G)=\left\{s x_{0}+t y_{0} \neq 0 \mid s, t \in K_{0} ; s / t \in C\right\}=T_{G}\left(x_{0}, y_{0}\right)
$$

is a circular cone in $E$, where $N=\left\{\left(x_{0}, y_{0}\right)\right\}$ and $G\left(x_{0}, y_{0}\right)=C$. Now define an a.h.p. $P \in \mathbf{P}_{3}$ by

$$
P(x) \equiv P\left(s x_{0}+t y_{0}\right)=s^{3}+s^{2} t+s t^{2}+t^{3}=(s+t)\left(s^{2}+t^{2}\right)
$$

for every $x=(s, t) \in E$. Obviously $Z_{P}\left(x_{0}, y_{0}\right) \subseteq T_{G}\left(x_{0}, y_{0}\right)$ as $s^{2}+t^{2}$ cannot vanish unless $s=t=0$ (cf. [1, p. 36]). Therefore, the hypotheses of Theorem (3.1) are satisfied by $P$ and $E_{0}(N, G)$. Given $x=(s, t)$ and $x_{1}=\left(s_{1}, t_{1}\right)$, we see [9, equation (2.4)] that

$$
\begin{aligned}
P\left(x_{1}, x, \ldots, x\right) & =(1 / 3) \cdot\left[s_{1} \partial P / \partial s+t_{1} \partial P / \partial t\right] \\
& =(1 / 3) \cdot\left[s_{1}\left(3 s^{2}+2 s t+t^{2}\right)+t_{1}\left(3 t^{2}+2 s t+s^{2}\right)\right]
\end{aligned}
$$

If we choose $x=(1+\sqrt{6}, 1)$ and $x_{1}=(1,-2)$, then $x$ and $x_{1}$ do not belong to $E_{0}(N, G)$ whereas $P\left(x_{1}, x, \ldots, x\right)=0$.

Now we give another example to show that Theorem (3.1) and its Corollaries (3.3) and (3.4) are best possible in the sense that the generalized circular regions $G(x, y)$ or $C$ (used in those results) cannot be replaced, in general, by generalized circular regions adjoined with arbitrary subsets of their boundary.

ExAMPLE (3.6). Take $E=\mathbf{C}^{2}, K=\mathbf{C}$, and $C=\{z \in \mathbf{C} \mid \operatorname{Im}(z)<0\} \cup\{1,2\}$. Then $C \notin D\left(\mathrm{C}_{\omega}\right)$, but the interior of $C$ does belong to $D\left(\mathbf{C}_{\omega}\right)$. If we take $x_{0}=$ $(1,0), y_{0}=(0,1), N=\left\{\left(x_{0}, y_{0}\right)\right\}$ and define $G\left(x_{0}, y_{0}\right)=C$, then $E_{0}(N, G)=$ $\left\{s x_{0}+t y_{0} \neq 0 \mid s, t \in \mathrm{C} ; s / t \in C\right\}=T_{G}\left(x_{0}, y_{0}\right)$ is a circular cone in $E$. Next, we define an a.h.p. $P \in \mathrm{P}_{2}$ from $\mathrm{C}^{2}$ to $\mathrm{C}$ by 


$$
P(x) \equiv P\left(s x_{0}+t y_{0}\right)=2 t^{2}-3 s t+s^{2}=(s-t)(s-2 t),
$$

for all $x=(s, t) \in \mathrm{C}^{2}$. Then $Z_{P}\left(x_{0}, y_{0}\right) \subseteq T_{G}\left(x_{0}, y_{0}\right)$. If $x_{1}=\left(s_{1}, t_{1}\right)$, then $[8$, p. 45]

$$
\begin{aligned}
P\left(x_{1}, x, \ldots, x\right) & =(1 / 2) \cdot\left(s_{1} \partial P / \partial s+t_{1} \partial P / \partial t\right), \\
& =(1 / 2) \cdot\left[s_{1}(2 s-3 t)+t_{1}(4 t-3 s)\right] .
\end{aligned}
$$

Choosing $x=(4,3)$ and $x_{1}=(0,1)$, we notice that $x, x_{1} \notin E_{0}(N, G)$ whereas $P\left(x_{1}, x, \ldots, x\right)=0$. This shows that Theorem (3.1) no longer holds for the type of sets $G(x, y)$. chosen above.

If we express the above facts in terms of an ordinary polynomial from $\mathbf{C}$ to $\mathrm{C}$, we obtain (see relations (3.4) and (3.5)) the following statement: If $f(z)$ $=z^{2}-3 z+2$, then all the zeros of $f$ lie in $C$ (as defined above) whereas $f_{1}\left(\zeta_{1}, z\right)=0$ for $\zeta_{1}=0$ and $z=4 / 3$ (both being outside $C$ ). This shows that Corollaries (3.3) and (3.4) do not hold in general for the type of sets $C$ considered above.

In the following examples and succeeding remarks, we show that there exist polynomials and circular cones (hermitian, or otherwise) satisfying the hypotheses of Theorem (3.1). This fact, together with Theorem (2.4), establishes that Theorem (3.1) is a strengthened generalization of Hörmander's theorem [6, Lemma 2].

Example (3.7). Let $H$ be a Hamel basis for a (finite or infinite-dimensional) vector space $E$ over $K$. Then every element $x$ of $E$ can be uniquely written (except for the order of its terms) as a finite linear combination of elements of $H$. (The proof is similar to the one given by Wilansky [14, Theorem 1, p. 15] for the case when $K=$ C.) We write $x=\Sigma \alpha \cdot h$ ( $h$ being in $H$ and $\alpha$ in $K$ ) and, realizing that this means $\sum_{n=1}^{m} \alpha_{n} \cdot h_{n}$ for a certain finite set of scalars $\alpha_{n}$, we shall still understand that the summation $\Sigma \alpha \cdot h$ is carried over all of $H$ with zero coefficients for all but a finite number of elements $h$ in $H$. Given $x=\Sigma \alpha \cdot h$, we shall denote by $\Sigma \alpha$ the sum of all the coefficients of $h$ in the expression for $x$.

Given any nucleus $N$ of $E^{2}$, we take the circular cone $E_{0}(N, G)$, where $G$ is the circular mapping from $N$ to $D\left(K_{\omega}\right)$ defined as follows: For every $(x, y)$ $\in N$ (with $x=\Sigma \alpha \cdot h$ and $y=\Sigma \beta \cdot h$ ) we define

$$
\begin{array}{rlrl}
G(x, y) & =K_{\omega} \quad \text { if } \sum_{\alpha}=\sum_{\beta}=0, \\
& =\left\{-\sum_{\beta} / \sum_{\alpha}\right\}, & & \text { otherwise. }
\end{array}
$$

Therefore, in the two cases we respectively have 


$$
T_{G}(x, y)=\{s x+t y \neq 0 \mid s, t \in K\},
$$

and

$$
\begin{aligned}
T_{G}(x, y) & =\left\{s x+t y \neq 0\left|s, t \in K ; s / t=-\sum_{\beta}\right| \sum_{\alpha}\right\} \\
& =\left\{z \in L[x, y] \mid z=\sum_{\delta} \cdot h \neq 0 ; \sum_{\delta}=0\right\} .
\end{aligned}
$$

Consequently, the circular cone $E_{0}(N, G)$ is precisely given by

$$
E_{0}(N, G)=\left\{z \in E \mid z=\sum \delta \cdot h \neq 0 ; \sum_{\delta}=0\right\} .
$$

Now we define an $n$ th-degree a.h.p. $P$ from $E$ to $K$ by

$$
P(x)=\left(\sum_{\alpha}\right)^{n} \quad \forall x=\sum \alpha \cdot h \in E .
$$

Obviously then $Z_{P}(x, y) \subseteq T_{G}(x, y)$ for every $(x, y) \in N$. I.e., $P$ and $E_{0}(N, G)$ satisfy the hypotheses of Theorem (3.1).

EXAMPLE (3.8). In a two-dimensional vector space $E$ over $K$ with basis $\left\{x_{0}, y_{0}\right\}$, let us take any circular cone $E_{0}(N, G)$ given by (cf. Remark (2.1)) $E_{0}(N, G)=\left\{s x_{0}+t y_{0} \neq 0 \mid s, t \in K ; s / t \in C\right\}$, for some $C \in D\left(K_{\omega}\right)$, where $N=\left\{\left(x_{0}, y_{0}\right)\right\}$ and $G\left(x_{0}, y_{0}\right)=C$. If only a nonempty proper subset of the boundary of $C$ is included in $C$, we observe (as at the end of Example (2.3)) that the set $E_{0}(N, G)$ is not a hermitian cone. (Note that for $K=\mathrm{C}$, a choice of such circular cones $E_{0}(N, G)$ can easily be made by taking a generalized circular region $C$ in $D\left(\mathbf{C}_{\omega}\right)$ such that a proper nonempty connected subset of the boundary of $C$ is included in $C$.) Given such a circular cone $E_{0}(N, G)$ in $E$, we can always find an $n$ th-degree a.h.p. $P$ from $E$ to $K$ such that the null-set $Z_{P}\left(x_{0}, y_{0}\right)$ of $P$ is contained in $T_{G}\left(x_{0}, y_{0}\right)=E_{0}(N, G)$. We show this as follows: Take any elements $\rho_{1}, \ldots, \rho_{n}$ (one or more may possibly be on the boundary of $C$ ) of $C$ and define

$$
P(x) \equiv P\left(s x_{0}+t y_{0}\right)=\prod_{j=1}^{n}\left(s-\rho_{j} t\right) \quad \forall x=s x_{0}+t y_{0} \in E .
$$

Clearly, then $Z_{P}\left(x_{0}, y_{0}\right) \subseteq T_{G}\left(x_{0}, y_{0}\right)$.

Hence: For every circular cone (hermitian, or otherwise) in a two-dimensional vector space $E$ over $K$, we can always find an a.h.p. from $E$ to $K$ satisfying the hypotheses of Theorem (3.1).

ExAmple (3.9). As in Example (2.3), if $E_{1}=\{x \in E \mid x \neq 0 ; H(x, x)<0\}$ is a hermitian cone with $H$ as a hermitian symmetric form of Lorentz signature, then there exist linearly independent elements $x_{0}$ and $y_{0}$ such that $H\left(x_{0}, x_{0}\right)=$ $1, H\left(x_{0}, y_{0}\right)=0$, and $H\left(y_{0}, y_{0}\right)<0$. If we set $F=\left\{y \in E \mid H\left(x_{0}, y\right)=0\right\}$, then 
$H(y, y)<0$ for every $y$ in $F$ and any element $x$ of $E$ can be uniquely written (cf. [6, p. 58]) as $x=t x_{0}+y$, where $y \in F$ and $t=H\left(x, x_{0}\right)$. We can then define an $n$ th-degree a.h.p. $P$ from $E$ to $K$ by $P(x)=t^{n}$ for every $x$ in $E$. Following the construction of Example (2.3), we can take a circular cone $E_{0}\left(N, G_{0}\right)$ which is not a hermitian cone and which properly contains $E_{1}$. From the definition of $P$, we see that $P(x)=0$ if and only if $x \in F$. Since $F \subseteq E_{1}$ and $E_{1} \cap$ $L[x, y] \subseteq T_{G_{0}}(x, y)$, it then follows that $Z_{P}(x, y) \subseteq T_{G_{0}}(x, y)$ for every $(x, y)$ $\in N$. Hence: For every circular cone belonging to the class of circular cones discussed in the remark following Example (2.3), we can always find an a.h.p. satisfying the hypotheses of Theorem (3.1).

Remarks. Examples (3.7)-(3.9) show that there exist circular cones and a.h.p.'s satisfying the hypotheses of Theorem (3.1). In Examples (3.8) and (3.9), however, these cones may not necessarily be hermitian. Furthermore, Examples (3.8) and (3.9) exhibit a class of circular cones for which we can always find an ah.p. satisfying the hypotheses of Theorem (3.1). This class consists of the following type of circular cones:

(a) all circular cones (hermitian, or otherwise) in a two-dimensional vector space $E$ over $K$;

(b) all hermitian cones $E_{1}$ defined by a hermitian symmetric form of Lorentz signature;

(c) all circular cones $E_{0}(N, G)$ obtained via the construction in Example (2.3) (these are not hermitian cones-see remark following Example (2.3)).

4. A generalization of Grace's theorem. Before stating our theorem, we define the concept of apolarity (equivalent to the one given by Marden [10]) for a pair of a.h.p.'s, without introducing an additional operator [10, equation (2.2)]. Our definition is as follows: Let $P, Q$ be a.h.p.'s (both of degree $n$ ) given by

$$
\begin{aligned}
& P(s x+t y)=\sum_{k=0}^{n} C(n, k) \cdot A_{k}(x, y) \cdot s^{k} t^{n-k}, \\
& Q(s x+t y)=\sum_{k=0}^{n} C(n, k) \cdot B_{k}(x, y) \cdot s^{k} t^{n-k},
\end{aligned}
$$

where $C(n, k)=n ! / k !(n-k) !$. We say that $P$ and $Q$ are apolar with respect to (linearly independent) elements $x, y$ of $E$ (written briefly, $P \perp Q[x, y]$ ) if

$$
\sum_{k=0}^{n}(-1)^{k} C(n, k) A_{k}(x, y) B_{n-k}(x, y)=0 .
$$

Note that the above definition makes sense only when $x, y$ are linearly independent. For, otherwise, any two polynomials of the same degree turn out to be apolar as shown below: If $x$ and $y$ are nonzero linearly dependent elements of 
$E$ (of course $P, Q$ are apolar whenever $x$ or $y$ is zero), there exists a nonzero scalar $\alpha$ in $K$ such that $y=\infty x$. Therefore

$$
\begin{aligned}
& P(s x+t y)=(s+t \alpha)^{n} \cdot P(x)=\sum_{k=0}^{n} C(n, k) \alpha^{n-k} P(x) \cdot s^{k} t^{n-k} \\
& Q(s x+t y)=(s+t \alpha)^{n} \cdot Q(x)=\sum_{k=0}^{n} C(n, k) \alpha^{n-k} Q(x) \cdot s^{k} t^{n-k}
\end{aligned}
$$

That is, $A_{k}(x, y)=\alpha^{n-k} P(x), B_{k}(x, y)=\alpha^{n-k} Q(x)$, and

$$
\sum_{k=0}^{n}(-1)^{k} C(n, k) A_{k}(x, y) B_{n-k}(x, y)=\alpha^{n} \cdot P(x) \cdot Q(x) \cdot(1+(-1))^{n}=0 .
$$

Consequently $P \perp Q[x, y]$. That is why we shall assume (throughout our discussion of apolarity) that $x, y$ are linearly independent and that the dimension of $E$ is at least two.

Successive applications of Theorem (3.1) give the following lemma, which will be found useful in proving our main result.

Lemma (4.1). Let $P \in \mathrm{P}_{n}$ and $E_{0}(N, G)$ be a circular cone in $E$. If $Z_{P}(x, y) \subseteq T_{G}(x, y)$ for some $(x, y) \in N$, then $P\left(s_{1} x+t_{1} y, \ldots, s_{n} x+t_{n} y\right) \neq$ 0 for all nonzero elements $s_{j} x+t_{j} y \in L[x, y]-T_{G}(x, y), 1 \leqslant j \leqslant n$.

Proof. Applying Theorem (3.1) to the polynomial $P$ and the circular cone $T_{G}(x, y)$ in the subspace $L[x, y]$, we see that $P\left(x_{1}, x_{0}, \ldots, x_{0}\right) \neq 0$ for all nonzero elements $x_{1}, x_{0}$ in $L[x, y]-T_{G}(x, y)$. If we put

$$
P_{1}\left(x_{0}\right)=P\left(x_{1}, x_{0}, \ldots, x_{0}\right) \quad \forall x_{0} \in E,
$$

then $P_{1}$ is an $(n-1)$ th-degree a.h.p. such that $Z_{P_{1}}(x, y) \subseteq T_{G}(x, y)$. We conclude again from Theorem (3.1) that $P\left(x_{1}, x_{2}, x_{0}, \ldots, x_{0}\right) \neq 0$ for all nonzero elements $x_{1}, x_{2}, x_{0}$ in $L[x, y]-T_{G}(x, y)$. Continuing, similarly, we can prove the lemma under consideration.

Now we prove the following theorem, which generalizes the theorems of Marden [10, Theorem (3.1)], Zervos [16, p. 360], and Grace [8, Theorem (15.3)], [4].

Theorem (4.2). Let $E_{0}(N, G)$ be a circular cone in $E$ and $P, Q \in \mathbf{P}_{n}$ such that $Z_{P}(x, y) \subseteq T_{G}(x, y)$ for some $(x, y) \in N$. If $P \perp Q[\xi, \eta]$ for some $\xi, \eta \in$ $L[x, y]$, then

$$
Z_{Q}(x, y) \cap E_{0}(N, G) \neq \varnothing .
$$

More precisely,

$$
Z_{Q}(x, y) \cap T_{G}(x, y) \neq \varnothing
$$


Proof. Note that statement (4.2) implies statement (4.1). Suppose, on the contrary, that the assertion (4.2) is false. Then $Z_{Q}(x, y) \subseteq L[x, y]-$ $T_{G}(x, y)$. Since $K$ is algebraically closed, we can write

$$
\begin{aligned}
& P(s \xi+t \eta)=\prod_{j=1}^{n}\left(\delta_{j} s-\gamma_{j} t\right)=\sum_{k=0}^{n} C(n, k) A_{k} s^{k} t^{n-k}, \\
& Q(s \xi+t \eta)=\prod_{j=1}^{n}\left(\beta_{j} s-\alpha_{j} t\right)=\sum_{k=0}^{n} C(n, k) B_{k} s^{k} t^{n-k},
\end{aligned}
$$

where the coefficients $\alpha_{j}, \beta_{j}, \gamma_{j}, \delta_{j}, A_{k}, B_{k}$ are all elements of $K$ which depend only on $\xi, \eta$ and are independent of $s$ and $t$. Obviously, since $L[x, y]=L[\xi, \eta]$, $Z_{P}(x, y)=Z_{P}(\xi, \eta)$, and $Z_{Q}(x, y)=Z_{Q}(\xi, \eta)$, we notice that $\alpha_{j} \xi+\beta_{j} \eta$ are all nonzero elements in $L[x, y]-T_{G}(x, y)$. By Lemma (4.1) then

$$
P\left(\alpha_{1} \xi+\beta_{1} \eta, \ldots, \alpha_{n} \xi+\beta_{n} \eta\right) \neq 0 \text {. }
$$

Let $S_{k}$ denote the sum of all possible products of the form $\beta_{1} \cdots \beta_{k} \cdot \alpha_{k+1}$ $\ldots \alpha_{n}$ got by permuting $1,2, \ldots, n$ in all possible ways. Then the definition of polar gives

$$
P\left(\alpha_{1} \xi+\beta_{1} \eta, \ldots, \alpha_{n} \xi+\beta_{n} \eta\right)=\sum_{k=0}^{n} s_{n-k} P\left(x_{1}, \ldots, x_{n}\right),
$$

where $x_{j}=\xi$ for $j \leqslant k$ and $x_{j}=\eta$ for $j>k$. Comparing the expressions for $Q(s \xi+t \eta)$, we get

$$
C(n, k) \cdot B_{k}=(-1)^{n-k} S_{k}
$$

Since

$$
\begin{aligned}
P(s \xi+t \eta) & =P(s \xi+t \eta, \ldots, s \xi+t \eta) \\
& =\sum_{k=0}^{n} C(n, k) \cdot P\left(x_{1}, \ldots, x_{n}\right) \cdot s^{k} t^{n-k}
\end{aligned}
$$

where $x_{j}=\xi$ for $j \leqslant k$ and $x_{j}=\eta$ for $j>k$, we obtain

$$
A_{k}=P\left(x_{1}, \ldots, x_{n}\right) \text { for all } k \text {. }
$$

Consequently, the assertions (4.3)-(4.6) contradict the fact that

$$
\sum_{k=0}^{n}(-1)^{k} C(n, k) \cdot A_{k} B_{n-k}=0 \text {. }
$$

This completes the proof of Theorem (4.2).

First specialization of this theorem to hermitian cones gives the following corollary, a result due to Marden [10, Theorem (3.1)].

Corollary (4.3). Let $E_{1}=\{x \in E \mid x \neq 0 ; H(x, x) \leqslant 0\}$ be a hermitian 
cone in $E$, where $H$ is a hermitian symmetric form defined from $E^{2}$ to $K$. Let $P, Q \in \mathrm{P}_{n}$ such that $P \perp Q[x, y]$ for some $x, y$ in $E$. If $Z_{P}(x, y) \subseteq E_{1}$, then $Z_{Q}(x, y) \cap E_{1} \neq \varnothing$.

Proof. We take a nucleus $N$ such that $(x, y) \in N$ (this is always possible). Proposition (2.2) then implies the existence of a circular mapping $G$ such that $E_{0}(N, G)=E_{1}$ and $E_{1} \cap L\left[x_{0}, y_{0}\right]=T_{G}\left(x_{0}, y_{0}\right)$ for every $\left(x_{0}, y_{0}\right) \in N$. Consequently, $Z_{P}(x, y) \subseteq T_{G}(x, y)$ and $P \perp Q[x, y]$. The proof is now complete in view of Theorem (4.2).

Our second application of Theorem (4.2) gives the following corollary, an improvement upon Grace's theorem [8, Theorem (15.3)] in the sense that we use generalized circular regions instead of the classical ones.

COROllary (4.4). Let $\mathrm{C}$ denote the field of complex numbers and let $f$, $\mathrm{g}$ be nth-degree polynomials (from $\mathbf{C}$ to $\mathbf{C}$ ) given by

$$
f(z)=\sum_{k=0}^{n} C(n, k) A_{k} z^{k}, g(z)=\sum_{k=0}^{n} C(n, k) B_{k} z^{k} .
$$

If $f$ and $g$ are apolar, i.e. $\Sigma_{k=0}^{n}(-1)^{k} C(n, k) A_{k} B_{n-k}=0$, and if all the zeros of $f$ lie in a generalized circular region $C$ of $C_{\omega}$, then at least one zero of $g$ lies in $C$.

Proof. Proceeding as in the proof of Corollary (3.3) and using the same notations, let us take $N=\left\{\left(x_{0}, y_{0}\right)\right\}, G\left(x_{0}, y_{0}\right)=C$, and define

$$
\begin{aligned}
& E_{0}(N, G)=\left\{s x_{0}+t y_{0} \neq 0 \mid s, t \in \mathrm{C} ; s / t \in C\right\}=T_{G}\left(x_{0}, y_{0}\right), \\
& P(x) \equiv P\left(s x_{0}+t y_{0}\right)=\sum_{k=0}^{n} C(n, k) A_{k} s^{k} t^{n-k} \quad \forall x=(s, t) \in \mathbf{C}^{2}, \\
& Q(x) \equiv Q\left(s x_{0}+t y_{0}\right)=\sum_{k=0}^{n} C(n, k) B_{k} s^{k} t^{n-k} \quad \forall x=(s, t) \in \mathrm{C}^{2} .
\end{aligned}
$$

Then $P(x)=t^{n} \cdot f(s / t)$ and $Q(x)=t^{n} \cdot g(s / t)$ for all nonzero elements $x=(s, t)$ $\in \mathrm{C}^{2}$. Obviously $Z_{P}\left(x_{0}, y_{0}\right) \subseteq T_{G}\left(x_{0}, y_{0}\right)$ and $P \perp Q\left[x_{0}, y_{0}\right]$, where $\left(x_{0}, y_{0}\right)$ $\in N$. Now Theorem (4.2), applied with $(x, y)=\left(x_{0}, y_{0}\right)=(\xi, \eta)$, gives $Z_{Q}\left(x_{0}, y_{0}\right) \cap T_{G}\left(x_{0}, y_{0}\right) \neq \varnothing$. Consequently, there exists a nonzero element $(s, t) \in C^{2}$ such that $g(s / t)=0, s / t \in G\left(x_{0}, y_{0}\right)$. I.e. $g(z)=0$ for some $z \in C$. This proves our corollary.

Last application of Theorem (4.2) gives the following result due to Zervos [16, p. 363]. In fact, Theorem (4.2) is a generalization of his result to vector spaces of any dimension (finite or infinite).

COROLlaRY (4.5). Let $f, g$ be nth-degree polynomials (from $K$ to $K$ ) defined by the expressions (4.7). If $f$ and $g$ are apolar and if all the zeros of $f$ lie 
in a generalized circular region $C$ of $K_{\omega}$, then $g$ has at least one zero in $C$.

Proof. The proof is same as that of Corollary (4.4) when $C$ is replaced by $K$.

In the remainder of this section, we discuss the validity of hypotheses and the degree of generality of Theorem (4.2).

It is known [8, p. 60] that there exist infinitely many polynomials (from C to $C)$ which are apolar to a given polynomial, and Szegö $\left(\left[11, \S 9\right.\right.$, Theorem $\left.1^{\prime}\right]$, see also $[8$, p. 61]) has given a method for constructing such polynomials. The following theorem presents such a construction for a.h.p.'s from $E$ to $K$.

Theorem (4.6). Given elements $x, y \in E$ and an a.h.p. $P \in P_{n}$, there exists an a.h.p. $Q^{*} \in \mathrm{P}_{n}$ such that $Q^{*} \perp P[x, y]$.

Proof. Let $P$ be given by

$$
P(s \xi+t \eta)=\sum_{k=0}^{n} C(n, k) A_{k}(\xi, \eta) s^{k} t^{n-k}
$$

and let us take any a.h.p. $Q \in \mathbf{P}_{n}$ given by

$$
\begin{aligned}
Q(s \xi+t \eta) & =\sum_{k=0}^{n} C(n, k) B_{k}(\xi, \eta) s^{k} t^{n-k} \\
& =\prod_{j=1}^{n}\left[\delta_{j}(\xi, \eta) \cdot s-\gamma_{j}(\xi, \eta) \cdot t\right] .
\end{aligned}
$$

Now define an a.h.p. $R \in \mathbf{P}_{2 n}$ by

$$
R(s \xi+t \eta)=\sum_{k=0}^{n}(-1)^{k} C(n, k) \cdot A_{k}(\xi, \eta) \cdot B_{k}(\xi, \eta) \cdot s^{2 k} t^{2(n-k)} .
$$

Finally, given the elements $x, y$ of $E$, we choose (there must exist one) an element $\mu x+\nu y$ in $Z_{R}(x, y)$ and define an a.h.p. $Q^{*} \in \mathrm{P}_{n}$ by

$$
\begin{aligned}
Q^{*}(s \xi+t \eta) & =\prod_{j=1}^{n}\left(\delta_{j}^{*}(\xi, \eta) \cdot s-\gamma_{j}^{*}(\xi, \eta) \cdot t\right) \\
& =\sum_{k=0}^{n} C(n, k) B_{k}^{*}(\xi, \eta) s^{k} t^{n-k} \quad \text { (say), }
\end{aligned}
$$

where $\delta_{j}^{*}(\xi, \eta)=\nu^{2} \gamma_{j}(\xi, \eta)$ and $\gamma_{j}^{*}(\xi, \eta)=\mu^{2} \delta_{j}(\xi, \eta)$. We claim that $Q^{*} \perp P[x, y]$ as follows: For convenience of notations, we shall write $A_{k}(x, y), B_{k}(x, y)$, $B_{k}^{*}(x, y), \delta_{j}(x, y)$, and $\gamma_{j}(x, y)$ simply as $A_{k}, B_{k}, B_{k}^{*}, \delta_{j}$, and $\gamma_{j}$ respectively. So that $\delta_{j}^{*}=\nu^{2} \cdot \gamma_{j}$ and $\gamma_{j}^{*}=\mu^{2} \cdot \delta_{j}$. Let $S_{k}$ (resp. $S_{k}^{*}$ ) denote the sum of all possible products of the form $\delta_{1} \cdots \delta_{k} \cdot \gamma_{k+1} \cdots \gamma_{n}$ (resp. $\delta_{1}^{*} \cdots \delta_{k}^{*} \cdot$ $\gamma_{k+1}^{*} \cdots \gamma_{n}^{*}$ ) got by permuting $1,2, \ldots, n$ in all possible ways. Then

$$
S_{k}^{*}=\nu^{2 k} \mu^{2(n-k)} \cdot S_{n-k} \text {. }
$$


But the expressions for $Q^{*}(s x+t y)$ and $Q(s x+t y)$ respectively imply that

$$
(-1)^{n-k} S_{k}^{*}=C(n, k) B_{k}^{*} \text { and }(-1)^{n-k} S_{k}=C(n, k) B_{k}
$$

Therefore

$$
B_{k}^{*}=(-1)^{n} \cdot \nu^{2 k} \mu^{2(n-k)} \cdot B_{n-k}
$$

and

$$
\begin{aligned}
\sum_{k=0}^{n}(-1)^{k} C(n, k) A_{k} B_{n-k}^{*} & =(-1)^{n} \cdot \sum_{k=0}^{n}(-1)^{k} C(n, k) A_{k} B_{k} \mu^{2 k} \nu^{2(n-k)} \\
& =(-1)^{n} R(\mu x+\nu y)=0 .
\end{aligned}
$$

This establishes our claim.

The following example shows that Theorem (4.2) cannot be further generalized for vector spaces over nonalgebraically closed fields of characteristic zero.

EXAMPLE (4.7). Using the notations of Example (3.5) and taking the vector space $E\left(=K_{0}^{2}\right)$, the circular cone $E_{0}(N, G)$, and the a.h.p. $P \in \mathbf{P}_{3}$ of that example, let us define an a.h.p. $Q \in \mathbf{P}_{3}$ by

$$
Q(x) \equiv Q\left(s x_{0}+t y_{0}\right)=2 t^{3}+s t^{2}+10 s^{2} t+5 s^{3}=(s+2 t)\left(5 s^{2}+t^{2}\right)
$$

for every $x=(s, t) \in E$. Then $Z_{P}\left(x_{0}, y_{0}\right) \subseteq T_{G}\left(x_{0}, y_{0}\right)$, as shown in Example (3.5). Also, we can easily verify that $P \perp Q\left[x_{0}, y_{0}\right]$. Therefore $P, Q$, and $E_{0}(N, G)$ satisfy the hypotheses of Theorem $(4.2)$ when $(x, y)=\left(x_{0}, y_{0}\right)=$ $(\xi, \eta)$. Since $5 s^{2}+t^{2}$ cannot vanish unless $s=t=0$ (cf. [1, p. 36]), we notice that $Z_{Q}\left(x_{0}, y_{0}\right) \cap E_{0}(N, G)=\varnothing$, contrary to the conclusion of Theorem (4.2).

Next, we give another example to show that Theorem (4.2) and its Corollaries (4.4) and (4.5) are best possible in the following sense: The generalized circular regions $T_{G}(x, y)$ or $C$ (used in those results) cannot be replaced, in general, by generalized circular regions adjoined with arbitrary subsets of their boundary.

EXAMPLE (4.8). Let us take the vector space $E\left(=\mathrm{C}^{2}\right)$, the set $C$, the circular cone $E_{0}(N, G)$, and the 2nd-degree a.h.p. $P$ of Example (3.6) and define another 2nd-degree a.h.p. $Q$ from $\mathrm{C}^{2}$ to $\mathrm{C}$ by

$$
Q(x) \equiv Q\left(s x_{0}+t y_{0}\right)=-7 t^{2}-2 s t+5 s^{2}=(s+t)(5 s-7 t)
$$

for every $x=(s, t) \in \mathbf{C}^{2}$. Then it is trivial to see that $P \perp Q\left[x_{0}, y_{0}\right]$ and $Z_{P}\left(x_{0}, y_{0}\right) \subseteq T_{G}\left(x_{0}, y_{0}\right)$, whereas $Z_{Q}\left(x_{0}, y_{0}\right) \cap E_{0}(N, G)=\varnothing$. That is, Theorem (4.2) is no longer valid (in general) for the type of sets $G(x, y)$ considered.

If we express the above example in terms of ordinary polynomials (from C to C), we obtain (due to relation (3.4)) the following statement: If $f(z)=$ $z^{2}-3 z+2$ and $g(z)=5 z^{2}-2 z-7$, then $f$ and $g$ are apolar and all the zeros 
of $f$ lie in $C$, whereas no zero of $g$ lies in $C$. I.e., Corollaries (4.4) and (4.5) do not hold (in general) for the type of sets $C$ chosen here.

REMARK. In Examples (3.7)-(3.9) and the succeeding remarks, we have already shown the existence of ah.p.'s $P \in \mathrm{P}_{n}$ and circular cones $E_{0}(N, G)$ (hermitian, or otherwise) such that $Z_{P}(x, y) \subseteq T_{G}(x, y)$ for every $(x, y) \in N$. Consequently, in the light of Theorem (4.6), there exist apolar a.h.p.'s and circular cones (hermitian, or otherwise) satisfying the hypotheses of Theorem (4.2). This fact, together with Theorem (2.4), establishes that Theorem (4.2) is a strengthened generalization of Marden's theorem expressed in Corollary (4.3).

\section{REFERENCES}

1. N. Bourbaki, Eléments de mathématique. XIV. Part 1. Les structures fondamentales de l'analyse. Livre II: Algèbre. Chap. VI: Groupes et corps ordonnés, Actualitiés Sci. In dust., no. 1179, Hermann, Paris, 1952. MR 14, 237.

2. D. R. Curtiss, $A$ note on the preceding paper (Walsh [13]), Trans. Amer. Math. Soc. 24 (1922), 181-184.

3. J. Dieudonné, Sur le théorème de Grace et les relations algébriques analogues, Bull. Soc. Math. France 60 (1932), 173-196.

4. J. H. Grace, On the zeros of a polynomial, Proc. Cambridge Philos. Soc. 11 $(1900-1902), 352-357$.

5. E. Hille and R. S. Phillips, Functional analysis and semi-groups, rev. ed., Amer. Math. Soc. Colloq. Publ., vol. 31, Amer. Math. Soc., Providence, R. I., 1957. MR 19, 664. 27.

6. L. Hörmander, On a theorem of Grace, Math. Scand. 2 (1954), 55-64. MR 16,

7. E. Laguerre, Oeuvres 1, Paris, 1898.

8. M. Marden, Geometry of polynomials, rev. ed., Math. Surveys, no. 3, Amer. Math. Soc., Providence, R. I., 1966.

9. - A generalization of a theorem of Bôcher, SIAM J. Numer. Anal. 3 (1966), 269-275. MR 34 \#1496.

10. On composite abstract homogeneous polynomials, Proc. Amer. Math. Soc. 22 (1969), 28-33. MR 40 \#4427.

11. G. Szegö, Bemerkungen zu einem Satz von J. H. Grace über die Wurzein algebraischer Gleichungen, Math. Z. 13 (1922), 28-55.

12. B. L. van der Waerden, Algebra. Vol. I, 4th ed., Die Grundlehren der math. Wissenschaften, Band 33, Springer-Verlag, Berlin, 1955; English transl., Ungar, New York, 1970.

13. J. L. Walsh, On the location of the roots of certain types of polynomials, Trans. Amer. Math. Soc. 24 (1922), 163-180.

14. A. Wilansky, Functional analysis, Blaisdell, New York, 1964. MR 30 \#425.

15. N. Zaheer, Null-sets of abstract homogeneous polynomials in vector spaces, Doctoral Thesis, Univ. of Wisconsin, Milwaukee, Wis., 1971.

16. S. Zervos, Aspects modernes de la localisation des zéros des polynômes d'une variable, Ann. Sci. Ecole Norm. Sup. (3) 77 (1960), 303-410. MR 23 \#A3241.

DEPARTMENT OF MATHEMATICS, ALIGARH MUSLIM UNIVERSITY, ALIGARH 202001, INDIA 